Chapter 10

\title{
Silicon Nitride Photonic Crystal Free-Standing Membranes: A Flexible Platform for Visible Spectral Range Devices
}

\author{
T. Stomeo, A. Qualtieri, F. Pisanello, L. Martiradonna, \\ P.P. Pompa, M. Grande, D'Orazio and M. De Vittorio
}

Additional information is available at the end of the chapter

http://dx.doi.org/10.5772/54846

\section{Introduction}

Two-dimensional (2D) photonic crystal (PhC) technology is well established at telecommunication bands, and materials such as Silicon (Si), Gallium Arsenide (GaAs) or Indium Phospide $(\mathrm{InP})$ represent a common solution for applications at these wavelengths [1-5]. However, the interest of scientific community on structures operating in other spectral regions, such as the visible one, is growing up for both linear and non- linear applications [6-8]. Indeed, 2D-PhC resonant cavities in the visible spectral range are considered a promising tool to boost photonic devices performance in several fields, such as biosensing, integrated optics, quantum communications, solar energy, etc. As a consequence of this wide area of interest, a photonic platform able to answer to the needs of all these fields would be attractive for scientific and technical communities.

Trying to develop such technological platform, the first problem one should face is the material choice. In principle it should be transparent in the whole visible spectral range with a relatively high refractive index (n), economical, compatible with silicon based technologies, robust, biocompatible and suited for easy functionalization with several biological species.

In past years several materials have been proposed with this purpose; among them, of remarkable interest are Gallium Nitride (GaN) [9], Gallium Phospide (GaP) [10], polymers [11] and Silicon Dioxide $\left(\mathrm{SiO}_{2}\right)[12,13]$. Another appealing material is Silicon Nitride $\left(\mathrm{Si}_{3} \mathrm{~N}_{4}\right)$, which answers to most of the above-mentioned requirements. Indeed, stoichiometric silicon nitride is transparent in the visible spectral range with a refractive index $\mathrm{n} \sim 1.9$ (@ $\lambda=600 \mathrm{~nm}$ ), it can be grown on Si with low-cost and widely diffused growth facilities such as Plasma Enhanced 
Chemical Vapor Deposition (PECVD) [14], it is compatible with Si based electronics (it is used as insulator in MOSFET gates $[15,16])$, it is biocompatible and can be functionalized with several kinds of proteins [17-19].

Several $\mathrm{Si}_{3} \mathrm{~N}_{4} 2 \mathrm{D}$-PhC cavities have been already proposed in past years [6,20,21], showing a maximum experimental quality factor $(Q)$ of $\sim 5000$ in the case of a double heterostructure nanocavity [21]. Moreover, recent advances in the development of nanobeam cavities have led to extremely high quality factors also in the visible range, with a maximum $Q$ of $\sim 55000$ [22-24].

This chapter is devoted to the use of $\mathrm{Si}_{3} \mathrm{~N}_{4} \mathrm{PhC}$ resonators as a flexible platform to realize photonic devices based on the engineering of nanoemitters spontaneous emission in the visible spectral range. First of all, the nanocavity design based on the closed band-gap principle will be presented and discussed. The chapter will then be focused on the nanotechnological procedures developed in recent years to realize high quality $\mathrm{Si}_{3} \mathrm{~N}_{4} \mathrm{PhC}$ cavities and the coupling of these structures with organic and inorganic nanoemitters. The versatility of the examined approaches will be also reviewed, showing how it is possible to couple several types of quantum light emitters to the two photonic states allowed in a closed band-gap single point defect nanocavity $[25,26]$. At the end of the chapter, a case of study on PhC-based biosensors [27] will be used to make the reader conscious of the possibility to realize advanced photonic devices in the visible spectral range exploiting the $\mathrm{Si}_{3} \mathrm{~N}_{4} \mathrm{PhC}$ technology. In the conclusions, we will discuss how improvements in modeling and processing of PhC structures in Silicon Nitride, which are highly compatible with both biological materials and inorganic quantum emitters, can further boost device performance, envisioning a broader application of twodimensional PhC nanocavities in the visible spectral range.

\section{Microcavity design: The closing band-gap and the modal selective tuning}

\subsection{The closing band-gap for low refractive index materials}

A system composed by a quantum light emitter coupled to a resonant optical mode can be modeled as two interacting oscillators. The strength of this interaction can lead to two different coupling regimes known as strong and weak coupling. In weak coupling regime, the free-space spontaneous emission rate $\left(\Gamma_{0}\right)$ is modified by the so-called Purcell effect: the coupled system emits with a rate $\Gamma_{\mathrm{C}}=\mathrm{F} \Gamma_{0}$, where $\mathrm{F}=3 /\left(4 \pi^{2}\right) \mathrm{Q} / \mathrm{V}(\lambda / \mathrm{n})^{3}$ is called Purcell Factor (Q and $\mathrm{V}$ are the quality factor and the modal volume of the photonic mode, respectively). When the system is instead in the strong coupling regime, the confined excitons and photons coherently exchange energy with a coupling strength, $g$, inversely proportional to $V$, i.e. $g \propto 1 / \sqrt{ } V$. Thus the properties of the photonic mode and, in particular, the electromagnetic field confinement in both time and spatial domains strongly affect the dynamic of the coupled system.

At visible wavelengths, these phenomena have been observed by means of several optically confined systems [28,29], but 2D-PhCs represent the most promising structures, since they give the best control on the optical properties of the resonators. To date, PhC cavities for visible spectral range are based on various geometries $[6,11,21]$ and on higher-order modes of the 
widely studied H1 defect [30] (sketched in Fig. 1(a), inset). This resonator consists of a missed hole in a triangular $\mathrm{PhC}$ lattice, and it allows two orthogonally polarized resonant modes in the photonic band gap (hereafter referred to as $\mathrm{x}$ - and $\mathrm{z}$-pole modes, on the base of the orientation of the wave vector).

The cross polarization of $x$ - and $z$-pole modes and the absence of higher-order states represent non-negligible advances for applications in quantum optics [31,34]. Moreover, the H1 cavity presents the lowest $\mathrm{V}$ among PhC point defects, thus enhancing quantum electrodynamic (QED) phenomena in both strongly and weakly coupled systems. However, obtaining small $\mathrm{V}$ at visible wavelengths is a challenging goal, because of the low refractive index of transparent materials in this spectral range, which reduces the effectiveness in localizing the optical modes. Nevertheless, the aforementioned advantages, together with the increasing interest toward the realization of efficient emitting devices in the visible spectral range, foster theoretical and experimental studies to find alternative routes to improve light confinement in low-index H1 systems.

In the following, we consider a resonator consisting of a point defect $\mathrm{H} 1$ in a triangular lattice of air holes (period $a$, radius $r$ ) realized in a silicon nitride slab having refractive index $\mathrm{n}=1.93$ and a thickness t. Plane-Wave Expansion (PWE) and 3D Finite Difference Time Domain (FDTD) algorithms [32] were used to investigate the electromagnetic response of such structure. All the calculations were restricted to modes with non-negligible components of the electric field along $\mathrm{x}$ and $\mathrm{z}$ and a non-negligible component of the magnetic field along $\mathrm{y}$ (hereafter referred to as TE- like modes).
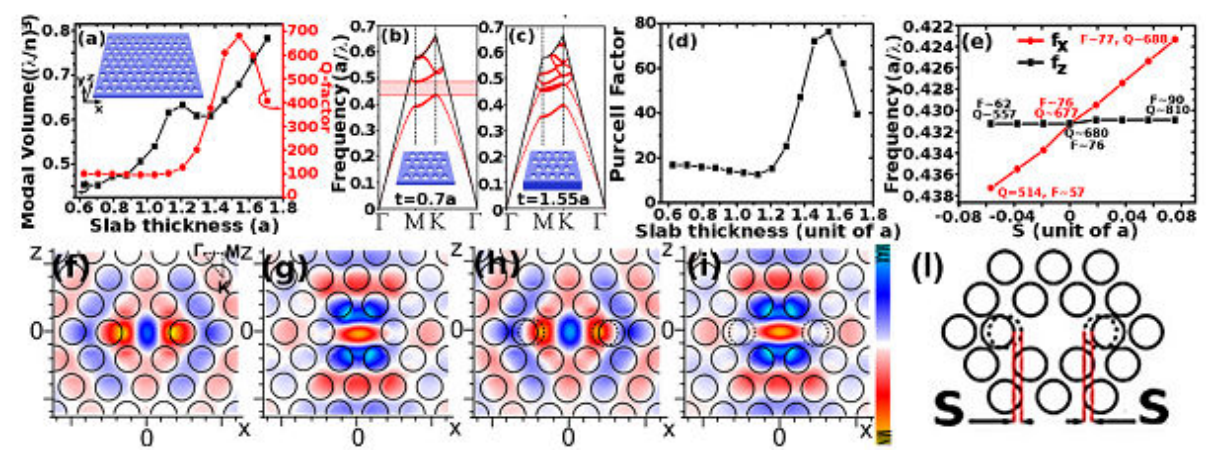

Figure 1. (a) Dependence of modal volume and Q factor on the thickness of the slab $t$. Inset, photonic crystal H1 cavity. (b), (c) Photonic band structure of the structure for $t=0.7 \mathrm{a}$ and $t=1.55 \mathrm{a}$, respectively. (d) Dependence of the Purcell factor on $\mathrm{t}($ for $\mathrm{S}=0$ ). (e) Modification of the resonant frequencies and of the $\mathrm{Q}$ factor of the degenerated modes when two cavity neighboring holes are moved, as shown in the inset. The holes are moved closer to (farther from) the center for $S<0(S>0)$. (f) $E_{z}$ for the x-pole mode in an unmodified $H 1$ cavity. (g) $E_{x}$ for the z-pole mode in an unmodified $\mathrm{H} 1$ cavity. (h) $E_{z}$ for the $x$-pole mode in a $H 1$ cavity with $S=0.2 a$. (i) $E_{x}$ for the z-pole mode in a $H 1$ cavity with $S=$ 0.2a. (I) Definition of S.

One way to realize ultrasmall-volume PhC cavities while keeping high Q- factors in the visible range and preserving the dipole-like shape of the modes is the so-called closing 
band-gap technique [33], involving PhC slab thickness ( $t$ ) optimization. As shown in Fig. $1(\mathrm{a})$, the $\mathrm{x}$-pole mode $\mathrm{Q}$-factor has a maximum for $t=1.55 a$, while it is almost constant for $t<1.2 a$. The Photonic Band-Gap (PBG) existing for $t=0.7 a$ disappears when $t$ is increased to 1.55a (see Figs. 1(b) and 1(c)). In agreement with the closing band-gap principle [33], this effect is assigned to a new nature of the electromagnetic confinement in the xz plane: it is not still due to the PBG, but it has to be assigned to the momentum space mismatch between the cavity mode and the second guided mode in the PhC slab. The increased thickness of the slab leads to slight variations of the $x$ - and $z$-pole modal profiles along $y$, thus leading to a wider modal volume, as shown in Fig. 1(a). However, these variations of $\mathrm{V}$ are negligible with respect to the increase in $\mathrm{Q}$, since the modal extension in the $\mathrm{xz}$ plane is preserved. Indeed the Purcell factor [Fig. 1(d)] follows the Qfactor behavior: for $t=1.55 a, \mathrm{~F}$ is maximized to $\mathrm{F} \sim 78$ with $\mathrm{V} \sim 0.68(\lambda / \mathrm{n})^{3}$ and $\mathrm{Q} \sim$ 700. A similar trend has been found for the z-pole mode.

\subsection{Modal selective tuning}

The $\mathrm{x}$ - and z-pole modes engineering would foster many applications based on $\mathrm{H} 1$ nanocavities operating at visible wavelengths. For instance, the degeneracy of $\mathrm{x}$ - and z-pole modes may be useful for entangled photon generation [34]. Other applications, such as single-photon sources or PhC-based optical read out of lab-on-chip devices [27], require well-defined and linearly polarized non-degenerate resonances. Several solutions have been reported in past years to break the energy degeneracy of the optical modes or to recover it [30,35-37]. A promising strategy to obtain a control on $\mathrm{x}$ - and z-pole modes is displayed in Fig. 1(e): by acting on two cavity neighboring holes, the resonant frequency of the $x$-pole mode $\left(f_{x}\right)$ can be significantly modified while keeping constant the z-pole mode one. This finding can be ascribed to the selective modification of the wavevector $k=\left(k_{x}, k_{y}, k_{z}\right)$ along a specific axis. Indeed $\mathrm{x}$ - and $\mathrm{z}$-pole modes have the strongest component of $\mathrm{k}$ oriented along the $\mathrm{x}$ and $\mathrm{z}$ axes, respectively. If two holes are moved one toward each other along the $x$ axis $(S<0$, see Fig. 1(l) for definition), $k_{x}$ is modified without affecting $k_{z}$. As a consequence, $f_{x}$ increases while $f_{z}$ does not change. In the same way $f_{x}$ decreases for $S>0$, while keeping $f_{z}$ constant. Figures $1(f-i)$ display $x$ - and z-pole modal profiles for $S=0$ and $S=0.2 a$ : the electric field component along $x\left(E_{x}\right)$ of the z-pole mode profile remains unchanged when the holes are moved far from the center [Figs.1(g) and 1(i)]. The shift instead results in the elongation of the x-pole modal function along x [Figs. 1(f) and 1(h)], thus modifying its resonant frequency.

It is important to notice that such alterations of field distributions modify the modal $\mathrm{Q}$ factors [Fig. 1(e)]: when $S<0$, abrupt changes are introduced near the electric field maximum of the z-pole mode function, resulting in an increase in radiation losses and in a smaller $Q$ factor $(Q$ $\sim 557$ for $S=-0.057 a$ ) [38]. In contrast, if $S>0$ these abrupt variations are avoided, the radiative energy in the light-cone minimized, and the Q-factor of the z-pole mode enhanced together with almost preserved $V$ and $f_{z}$. The optimized $Q$-factor turns out to be $Q \sim 810$ for $S=0.075 a$, and the Purcell factor is assessed as F $\sim 90$.

These findings are confirmed by the analysis in the z-pole momentum space, obtained by using a 2D Fourier Transform (2DFT), reported in Figs $2(a)$ and (b) for $S=-0.1 a$ and $S=0.2 a$, respectively. 
The white circle of Figs 2 (a) and (b) delimits the leaky region, defined by the light cone [38-40]: the stronger the components within this area, the higher the radiation losses along $y$. For $S=-0.1 a$ (Figs. 2(a) and (e)) a sharp peak is present at the center of the leaky region, affecting the value of $Q$; instead if $S=0.2 a$ (Figs. 2 (b) and (f)), the 2DFT is almost constant inside the light cone.
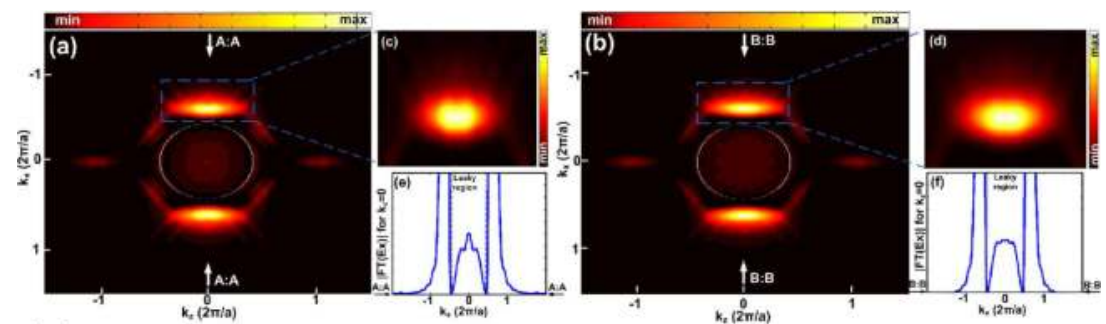

(g)

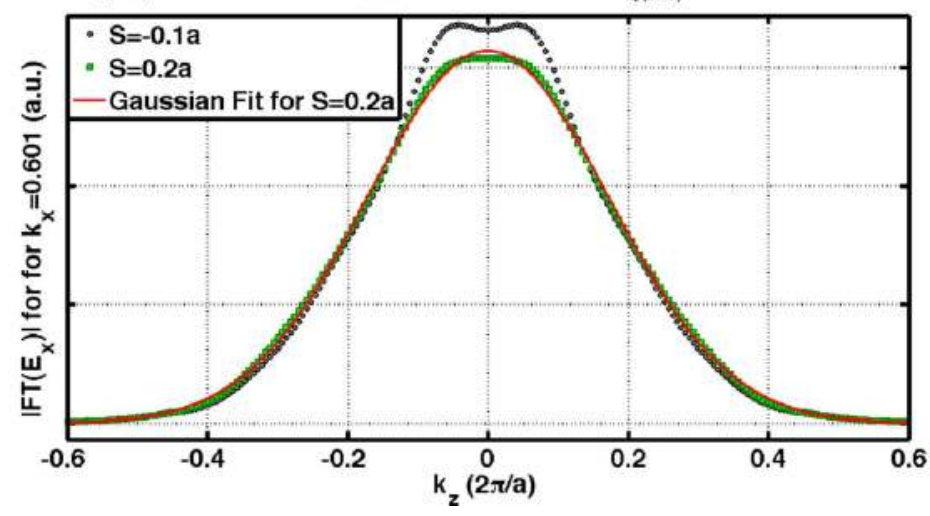

Figure 2. $E_{x}$ field-distribution in momentum space for the z-pole mode. The white circle represents the light-cone. (a) $\mid$ $2 \mathrm{DFT}\left(E_{x}\right) \mid$ for $S=-0.1 a$. (b) $\left|2 \mathrm{DFT}\left(E_{x}\right)\right|$ for a $S=0.2 a$. (c) Zoom to a specific area of (a). (d) Zoom to a specific area of (b). (e) Momentum function extracted from (a) for $k_{z}=0$. (f) Momentum function extracted from (b) for $k_{z}=0$. (g) Cross section of the lobe for $S=-0.1$ a shown in Fig. 2(c) (black circles) and of the lobe $S=0.2 a$ displayed in Fig. 2(d) (green squares). Red continuous line represents a gaussian fit of the case $S=0.2 a$

As demonstrated in [38], the Q-factor depends also on the shape of the two peaks outside the light cone. A Gaussian shape typically leads to higher Q-factor, giving a direct measure of the energy not coupled with the radiation mode. Figs. 2(c) and (d) show a zoom of Figs. 2(a) and (b), respectively: the behavior for $S=-0.1 a$ is far from a 2D Gaussian function. For $S=0.2 a$ (Fig. 2(d)), it is instead clear that by moving two holes far from the center, a 2D Gaussian function for these peaks is obtained, as also confirmed by the 1D gaussian fitting of the cross section of these lobes reported in Fig. 2(g). Therefore, by increasing the z-pole Q-factor, a positive $S$ does not substantially affect the position, modal volume and resonant frequency of the z-pole electric field main lobe, leading to a straightforward increase of the Purcell factor of microresonators.

This therefore verifies that momentum space engineering, a strategy exploited to improve the confinement of defect states localized within the PBG [11,38,42], can also be efficient for cavity resonances without PBG. 


\section{Fabrication of $\mathrm{Si}_{3} \mathrm{~N}_{4} \mathrm{PhC}$ nanocavities}

The 2D-PhC nanocavities were fabricated into a 400-nm-thick $\mathrm{Si}_{3} \mathrm{~N}_{4}$ layer deposited on a silicon substrate by means of Plasma Enhanced Chemical Vapor Deposition (PECVD) technique. $\mathrm{Si}_{3} \mathrm{~N}_{4}$ refractive index was measured through spectrophotometric methods (performed with a Varian Cary 5000 spectrophotometer), giving a value of $\mathrm{n}_{\mathrm{SiN}}=1.93 @ \lambda=600 \mathrm{~nm}$.

The PhC geometry was defined using a Raith150 e-beam lithography tool (equipped with a Gemini Column) operating at $30 \mathrm{kV}$. A thickness of $400 \mathrm{~nm}$ of ZEP520-A resist was chosen to ensure sufficient durability as a mask for pattern transfer into the underlying $\mathrm{Si}_{3} \mathrm{~N}_{4}$ and, at the same time, to ensure a good resolution of the e-beam writing. The key issue in the fabrication process is to achieve PhC devices with well-controlled patterns size.

A preliminary dose-test was performed to define the optimum layout since the actual size of the pattern is influenced by the electron dose. A proximity error correction (PEC) was also applied to accomplish this target and the final dose was determined through Scanning Electron Microscope (SEM) inspections at $10 \mathrm{kV}$. Moreover, in order to achieve smoother and circular holes and faster exposure, the EBL system was used in "circular mode". In this mode, every circular hole is exposed by the deflection of the beam along concentric circles.

The patterns defined in the ZEP were then transferred into the $\mathrm{Si}_{3} \mathrm{~N}_{4}$ layer using inductive coupled plasma reactive ion etching (ICP-RIE) in fluorine chemistry until the silicon substrate surface was reached. The membrane structure was released by wet etching of the underlying Si substrate in a TetraMethylAmmonium Hydroxide (TMAH) solution. Each sample consisted of an array of $\mathrm{H} 1$ nanocavities, whose dimensions were scaled according to the lattice period $a$ (in the range $a=257 \mathrm{~nm}-277 \mathrm{~nm}$ ) thus allowing spectral shifting of the resonant wavelength. Fig. 3(a) shows a Scanning Electron Microscope (SEM) image of the realized resonators.
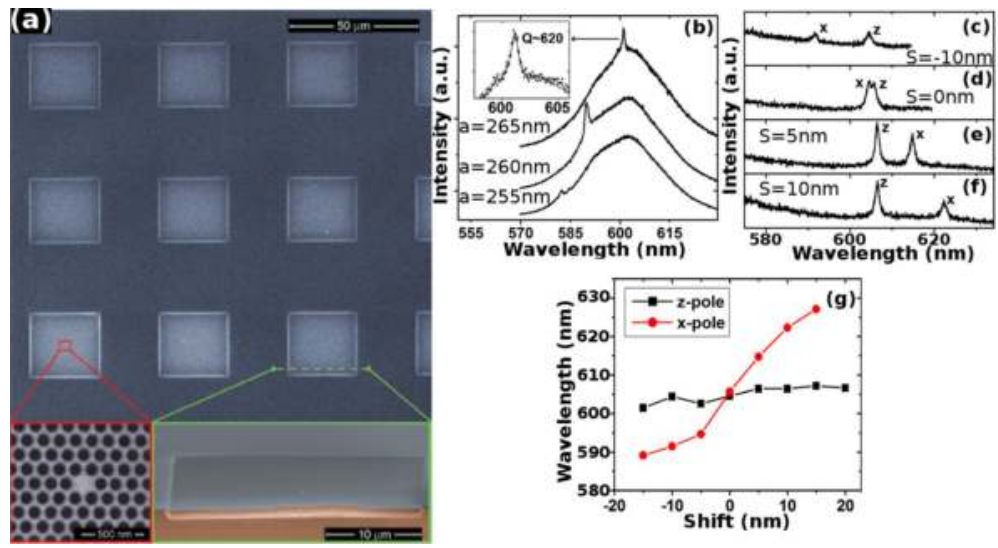

Figure 3. a) Top and bird's eyes view of the realized nanocavities acquired by SEM. (b) Resonances obtained by drop casting colloidal nanocrystals on the structure for three different values of $a$ and $r=0.308 a$. The inset shows the Lorentzian fitting of the resonant peak for $a=265 \mathrm{~nm}$. (c)-(f) Resonance of $x$ - and $z$-pole modes for different values of the hole shift and $a=270 \mathrm{~nm}$. (g) Resonant frequencies of $x$ - and z-pole modes as a function of the hole shift. 


\section{Coupling of H1 nanocavity with organics and inorganics emitters}

The theoretical findings described in section 2 have been experimentally demonstrated by coupling to the nanocavities both cyanine 3 (Cy3) fluorophore and core/shell CdSe/CdS colloidal dot-in-rod (DR) nanocrystals. Room temperature microphotoluminescence ( $\mu \mathrm{PL})$ characterization was therefore performed to investigate the properties of the coupled system.

\subsection{Nanoemitters deposited on top of the cavity}

A micromolar solution $\left(10^{-6} \mathrm{~mol} / \mathrm{l}\right)$ of DRs in toluene was prepared by using the synthesis procedure described in [43] by L. Carbone and co-workers and drop-casted on the realized microcavities. Figure 3(b) displays three resonances for three different values of $a$. The resonant peaks are well fitted by a Lorentzian function [Fig. 3(b), inset] and result in a maximum $\mathrm{Q} \sim$ 620 for an unmodified H1 cavity ( $a \sim 265 \mathrm{~nm})$.

To explore the mode shifting over a wide spectral range, an organic fluorophore (Cy3) with broad emission spectrum was immobilized on the device. The $\mu$ PL spectra for different values of $\mathrm{S}$ reported in Figures 3(c-f) show that the z-pole mode is almost unaffected by holes shifting, while x-pole resonant wavelength can be broadly tuned by means of $S$. Polarization-resolved measurements were carried out to identify the two modes, and their resonant wavelengths $\left(\lambda_{x}\right.$ and $\left.\lambda_{z}\right)$ as a function of $S$ are displayed in Fig. 3(g). In agreement with the theoretical results of Fig. 3(e), the x-pole mode is tunable over a range $\Delta \lambda_{\mathrm{x}} \sim 40 \mathrm{~nm}$. Small discrepancies between experimental results and theoretical calculations have been observed in terms of slight variations of $\lambda_{z}$ and weak nonlinearity of $\lambda_{x}$; since these variations do not show a clear dependence on $S$, they could be reasonably attributed to unavoidable fabrication imperfections. The theoretical findings about the influence of the holes position on the z-pole Q-factor have been confirmed by the experiments. For $S=15 \mathrm{~nm} Q \sim 750$ has been measured, while for $\mathrm{S}=-20 \mathrm{~nm}$ the z-pole $\mathrm{Q}$-factor falls down to a value of $\sim 200$.

\subsection{Colloidal nanocrystals localized in the maximum of the electric field distribution}

It is well known that in 2D-PhC slabs the in-plane confinement $(x z)$ is due to the photonic band gap produced by the PhC periodicity, while in the out-of-plane direction (y) the confinement is due to the total internal reflection. As already mentioned, in the xz plane the electromagnetic field is localized in the center of the cavity (see figures 1(f-i)); FDTD simulations show also that along $y$ the main lobe of the confined radiation is in the center of the slab (see Fig. 4).

The coupling reported in section 4.1 is thus not optimized, as the nanocrystals and the organic molecules are deposited on top of the cavities.

A viable strategy to approach the maximum allowed Purcell factor is to localize the nanoemitters in the center of the slab. This has been done with colloidal dot-in-rod nanocrystals using the same fabrication process described in section 3 and splitting the growth procedure of the $\mathrm{Si}_{3} \mathrm{~N}_{4}$ slab in two steps. Figure 5(a) shows a sketch of the fabrication procedure. First of all, a $200 \mathrm{~nm}$ thick $\mathrm{Si}_{3} \mathrm{~N}_{4}$ slab was grown on a Si substrate. A thin layer of colloidal DRs, with a molar concentration of $\sim 10^{-6} \mathrm{~mol} / \mathrm{l}$ was then spin-coat- 
ed on it with a rotating speed of $500 \mathrm{rpm}$, thus obtaining a thickness lower than $10 \mathrm{~nm}$ as assessed by SEM inspection. After solvent evaporation, a second $200 \mathrm{~nm}$ thick layer of $\mathrm{Si}_{3} \mathrm{~N}_{4}$ was grown on top of the sample. We verified the uniformity of the deposited layer by exploiting both the morphological characterization and the photoluminescence maps collected by a confocal microscope. A SEM cross-section of the resulting sandwiched structure is shown in Fig. 5(b). The nanocavities were then realized through electron beam lithography and dry and wet etching processes by following exactly the fabrication procedure reported in paragraph 3 (Fig. 5(c)).

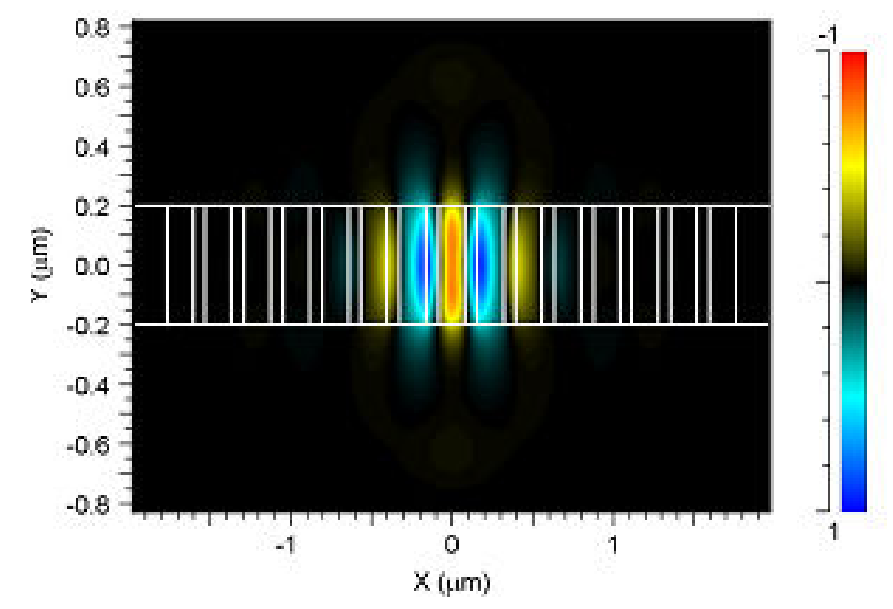

Figure 4. Cross view of the electric field $x$-component with the superposition of the slab cross-section at $z=0$.

Also in this case, the optical measurements of the nanocavities were carried out by the OLYMPUS FluoView 1000 confocal laser scanning microscope, with a spatial resolution of 200 $\mathrm{nm}$. A CW laser diode emitting at wavelength $\lambda_{\mathrm{ex}}=405 \mathrm{~nm}$ was used as excitation source. In Fig. 5(d) are reported the photoluminescence spectra collected from the 2D-PhC H1 nanocavities with different lattice constants $a$. Superimposed to the broad emission spectrum (FWHM $\sim 30 \mathrm{~nm}$ ) of NCs uncoupled to the cavity, sharp peaks with a quality factor of about 600 are clearly detected, assessing the modulating effects of the PhC nanocavity on the emission of NCs coupled to the optical mode localized in the defect. Moreover, the normalized frequency $\mathrm{a} / \lambda$ of the experimental results was found to be about $\mathrm{a} / \lambda \sim 0.46$ against the expected value of $\mathrm{a} / \lambda$ of $\sim 0.431$. As already suggested in case of the modal selective tuning, this slight difference can be mainly attributed to the effects of fabrication imperfections, inducing unavoidable uncontrolled variations in the optical properties of the PhC nanocavities [45].

The efficient coupling between the semiconductor nanocrystals layer and the dielectric cavity is due to the fact that the nanocrystals layer can be precisely positioned in the maximum of the confined electric field in the vertical direction. Indeed, in this case the Purcell effect results optimized [46] and the spontaneous emission rate strongly increased, leading to the 
possibility to measure a better Q-factor [47]. At the same time it is noteworthy to point out how the introduction of a guest material, embedded in two $\mathrm{Si}_{3} \mathrm{~N}_{4}$ layers, does not affect the optical properties of the nanocavity as shown by the good match of the calculated and measured Q-factors (equal to 680 and 600, respectively).

(a)

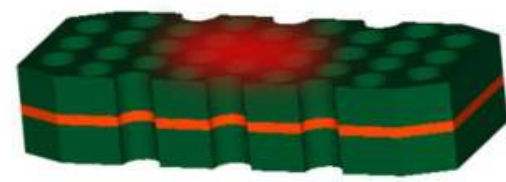

(c)

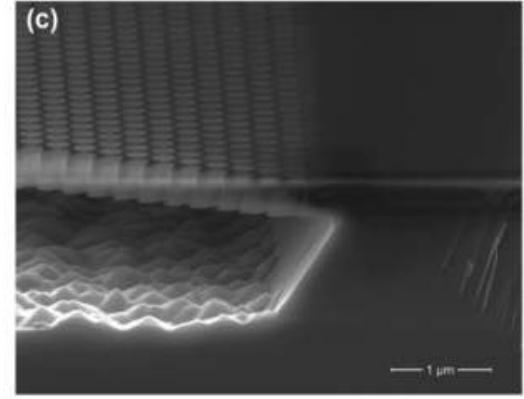

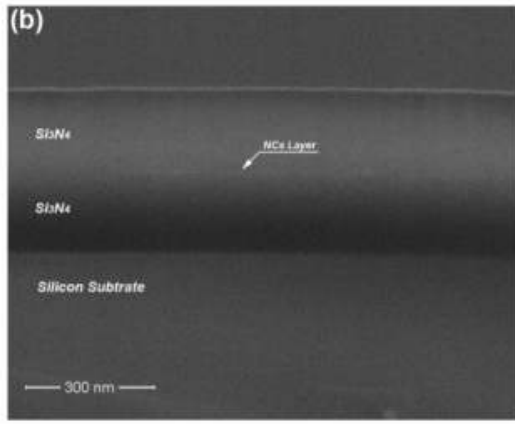

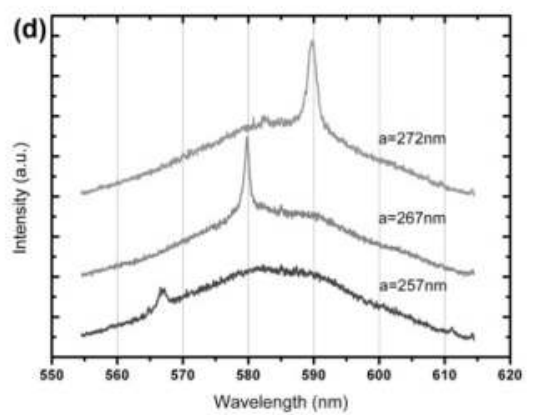

Figure 5. a) Sketch of the fabrication process, (b) cross-section SEM image of the un-patterned sample; (c) bird's eye view of the fabricated $\mathrm{Si}_{3} \mathrm{~N}_{4}$ 2D-PhC $\mathrm{H} 1$ nanocavity membrane and (d) photoluminescence spectra collected from the 2D-PhC H1 nanocavities with different lattice constants $a$.

\section{A biosensor based on $\mathrm{Si}_{3} \mathrm{~N}_{4} \mathrm{PhC}$ nanocavities}

Recently the light molding properties of $\mathrm{PhC}$ have been profitably exploited to boost the performance of optical sensors and transducers for biochemical analyses [48-50]. This paragraph proposes the idea of exploiting the sharp resonances of $\mathrm{PhC}$ nanocavities to assign unique spectral features to fluorophore-labeled bioanalytes, thus allowing their identification through wavelength-resolved light detection. Spectral tagging of organic dyes through photonic crystal nanocavities is experimentally proved to bring important benefits to cutting edge devices for biodiagnostics, such as DNA and protein biochips, in terms of improved sensitivity, efficiency and multiplexing capability. 


\subsection{Working principle}

PhC nanocavities can be embedded in a two-dimensional array, to realize an improved optical detection system of a miniaturized assay for genomic and proteomic analyses, (DNA or protein microarray). Fig. 6(a) is a three-dimensional sketch of the biochip architecture including different nanocavities, each having a different resonant wavelength. Moreover, a one-to-one correspondence is also preserved between a cavity and a group of specific bio-molecules (probes) immobilized on the surface (as shown in the expanded view of Fig. 6(a)). The asrealized chip can be exposed to a biological solution containing unknown target species, or analytes; conjugation between the analytes and their complementary probes takes place on the device surface [51]. Since the target analytes are typically labeled with fluorescent markers, the binding events can be revealed through optical inspection of the biochip readout area, thus allowing a complete compositional analysis of the assay [52].

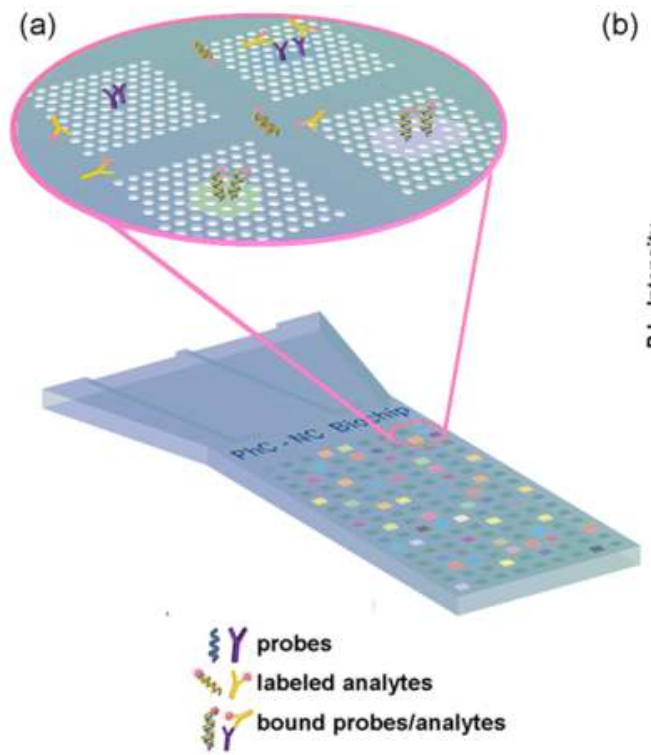

(b)

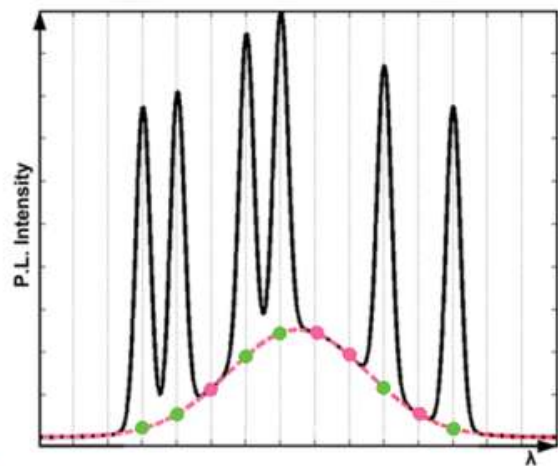

-- Marker luminescence

- Readout area luminescence

PhC tag detected

PhC tag not detected

Figure 6. Sketch of the proposed strategy for PhC-NC biochip. (a) Schematic of the array of PhC nanocavities patterned on the readout area. Inset: Detail showing examples of PhC nanocavities. The cavities are functionalized with different probes molecules, that specifically interact with complementary target analytes labeled with fluorescent markers. The signal is collected from this area and spectrally discriminated in order to identify the different spectral tags univocally associated to each nanocavity and thus to each bioprobe. (b) Example of a possible luminescence detected from the whole readout area (black line) as compared to the unmodified broad marker luminescence (red dotted line). The presence of each peak in the spectrum reveals the presence of the corresponding analyte in the investigated assay.

The recourse to a microarray configuration already allows the simultaneous analysis of a certain number of analytes thanks to spatial discrimination [52,53]. Here we upgrade the allowed degree of parallelization by assigning a peculiar spectral signature, given by the 
resonating behavior of each cavity, to each bioprobe immobilized on the surface. This gives the possibility to distinguish the spectral response of each target analyte bound to the corresponding probe, albeit a single common fluorophore is used for the labeling of the whole unknown solution. Fig. 6(b) exemplifies a possible spectral scan of the signal collected from the whole readout area. Different peaks can be observed on the emission spectrum of the fluorescent marker, each revealing the presence of a specific target analyte in the investigated assay. Besides the spatial discrimination implemented in microarray configurations, in this case the spectral distinction contributes substantially to the parallelization of the device. We also expect a beneficial effect given by Purcell effect, which increases the radiative emission rate of emitting materials interacting with quantum confined systems [54, 55]. Hence, a significant increase in the luminescence intensity of the markers coupled to the $\mathrm{PhC}$ cavities is envisioned, leading to a significant improvement of the signal-to-noise ratio and of the overall sensitivity of the biochip detection.

\subsection{Experimental results}

$\mathrm{PhC}$ nanocavities resonating in the visible spectral range were fabricated in $\mathrm{Si}_{3} \mathrm{~N}_{4}$ membranes on a Si substrate, exploiting the modified single defect $\mathrm{H} 1$ nanocavity described in section 2 $[33,43,47,57]$. Several chips were fabricated, each containing an array of optimized H1 resonators with variable lattice constant $a$, thus tuning the corresponding resonant wavelengths. We tested the proposed architecture both with single-stranded DNA (ss-DNA) and antibody probes immobilized on the $\mathrm{Si}_{3} \mathrm{~N}_{4}$ surface of two different devices. Complementary DNA targets or specific secondary antibodies, labeled with cyanine 3 (Су3) and rodhamine (TRITC) fluorophores, respectively, were then allowed to recognize the immobilized probes, thus obtaining a uniform fluorescent monolayer of the biomolecular species.

The effects of fluorescence enhancement and peak sharpening in resonant conditions are clearly observed in the emission spectra reported in Fig. 7(a) for PhC nanocavities treated with TRITC-labeled proteins and in Fig. 7(b) for DNA-functionalized nanocavities (five uppermost lines, compared to the lowest spectrum corresponding to the emission of Cy3- DNA strands without photonic resonators). In both cases it is evident that the change of the lattice period $a$ of the photonic crystal resonator leads to the modification of the spectral response coming from target analytes conjugated by the same broad emitting organic dye: a specific spectral feature is thus attributed to the target analytes captured on different cavities. The best measured Qfactor obtained in the PhC-nanocavities DNA-chip prototype is $\sim 725$, corresponding to a fullwidth at half maximum of $\sim 0.9 \mathrm{~nm}$. Taking into account the spectral resolution limits, a conservative estimate suggests the possibility to distinguish up to 150 different resonant peaks within the $150 \mathrm{~nm}$ bandwidth of the $\mathrm{Cy} 3$ emission spectrum. This means that up to 150 parallel analyses can be simultaneously performed with one single spectral scan of the readout area of the biochip, thus drastically decreasing the time required for a complete compositional identification.

By confocal microscopy it is also possible to visualize the effects of emission enhancement in resonant conditions, as reported in the photoluminescence maps reported in Fig. 7(c). In this array of five different nanocavities, functionalized with ss-DNA and hybridized with Cy3- 
labeled complementary DNA sequences we have performed a spectral scanning of the acquisition wavelength with a resolution of $2 \mathrm{~nm}$. When the detection wavelength matches one of the five resonating wavelengths of the nanocavities, marked from $\lambda_{1}$ to $\lambda_{5}$ in Fig. 7(b), it is possible to distinguish a bright spot in the center of each nanocavity.

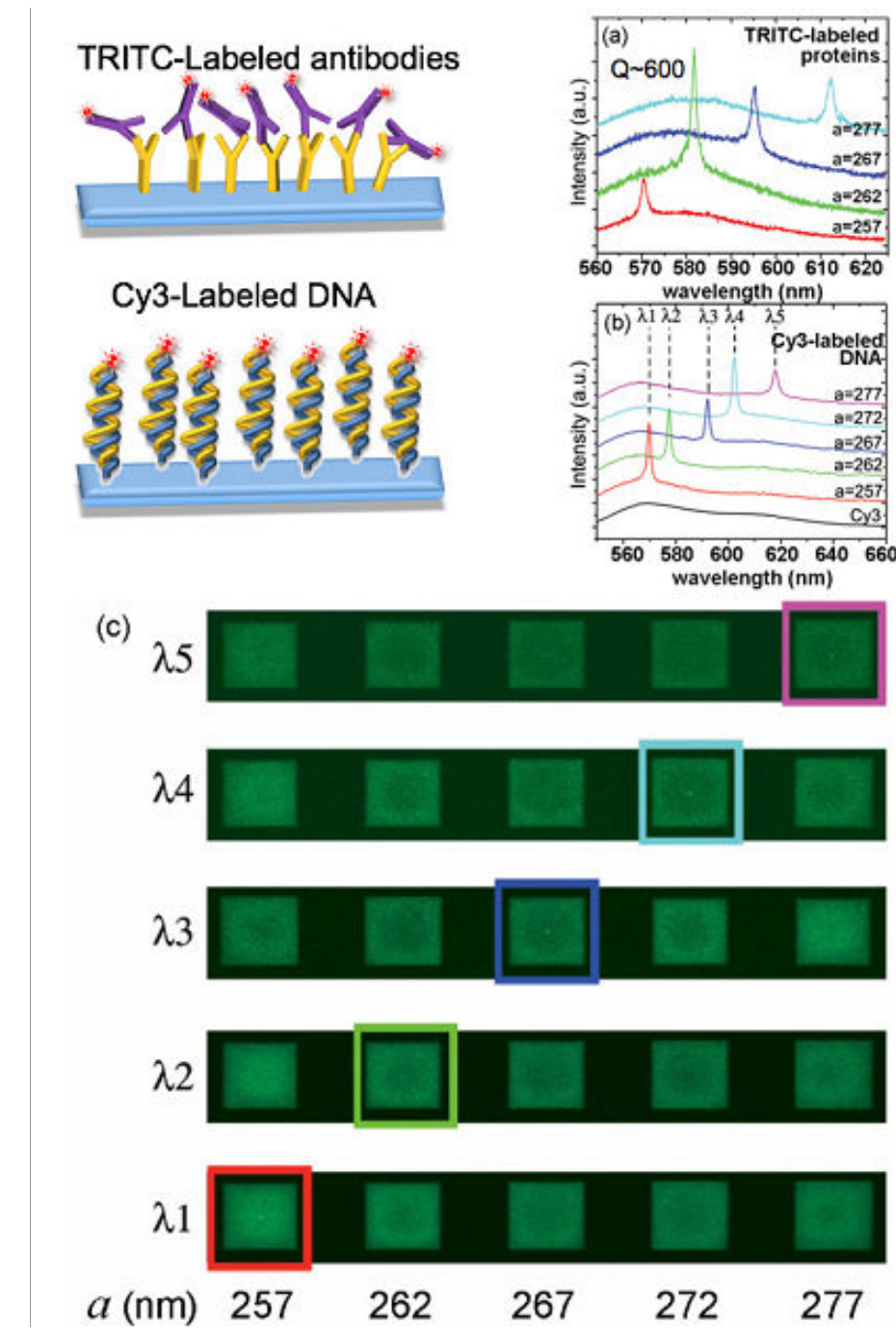

Figure 7. a) PL spectra collected from TRITC-labeled proteins captured onto the PhC nanocavities; (b) PL spectra collected from Cy3-labeled DNA (colored lines) onto the PhC nanocavities as compared to Cy3 emission spectrum collected on a PhC pattern (black line); (c) PL maps of an array of five Cye-labeled DNA-PhC nanocavities, collected at different wavelengths [also indicated in (b)]. For each spectrum and PL map, the reported a value indicates the lattice period of the measured PhC nanocavity. 
In order to quantify the enhancement effect of each photonic crystal pattern, in Fig. 8 it is reported a three-dimensional intensity profile collected on a Cy3-labeled DNA functionalized nanocavity in resonant conditions. The central bright spot corresponding to the H1 defect cavity reveals a signal improvement as high as 160 as compared to the luminescence coming from unpatterned $\mathrm{Si}_{3} \mathrm{~N}_{4}$ surface. A major role of the Purcell effect [54,55] can be envisioned, by virtue of the strong optical quantum confinement performed by the H1-shifted nanocavities.

Noteworthy, the photonic crystal pattern itself causes an improvement of fluorescence emission as compared to the surrounding unpatterned $\mathrm{Si}_{3} \mathrm{~N}_{4}$ layer, although the immobilization and hybridization processes have been homogeneously performed on the whole sample surface. In this case, an enhancement of $\sim 20$ times is achieved. This behavior may be ascribed to the combination of two effects. First, the free-standing membrane layer makes available a larger surface area to the probes immobilization (about a factor of 4 more than the unpatterned layer), resulting in a higher number of immobilized Cy3-labeled analytes in the $\mathrm{PhC}$ regions. Second, in 2DPhC patterns an efficient transfer channel between externally radiated light and energy trapped in the membrane is represented by the so-called leaky modes $[48,57,58]$. The coupling of such modes with the absorption or emission bands of neighboring emitters may lead to a significant increase of their luminescence. Although the photonic crystal pattern has not been specifically optimized to maximize such effect, the role of leaky modes localized on the PhC pattern for the further increase of the luminescence experimentally observed is not negligible.

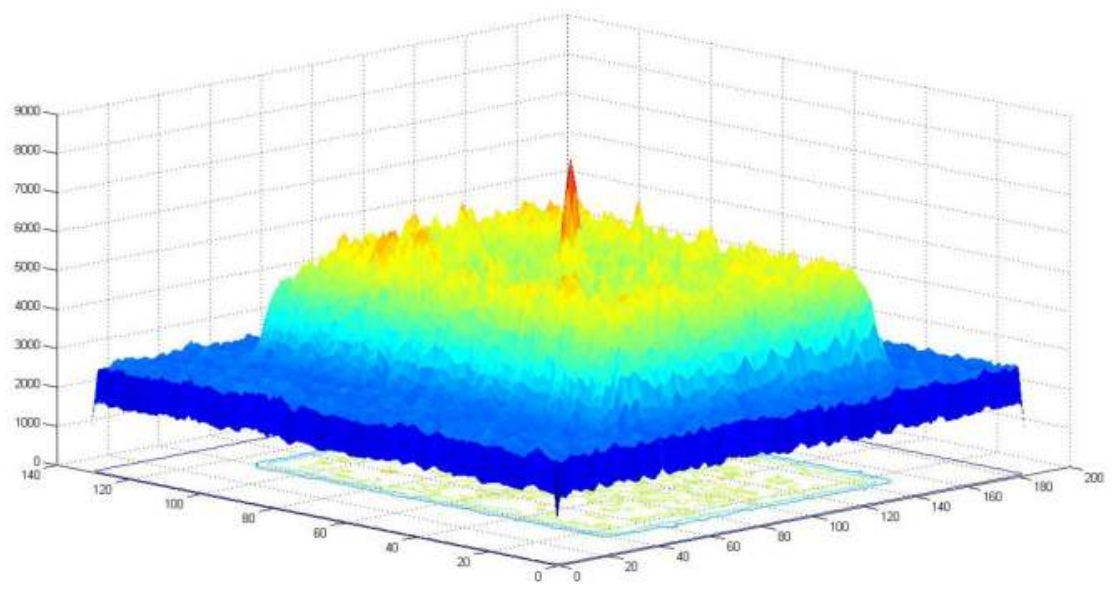

Figure 8. Three-dimensional intensity profile of photoluminescence collected from Cy3- labeled DNA captured by a functionalized nanocavity. Emission outside the PhC pattern has been normalized to unit. A 20-fold luminescence enhancement due to the PhC pattern, as compared to the unpatterned $\mathrm{Si}_{3} \mathrm{~N}_{4}$ surface, has been measured. The cavity confinement further enhances Cy3 emission up to 160-fold.

The insertion of $\mathrm{PhC}$ cavities in classical biochip architectures leads, therefore, to a huge increase of the emission intensity of fluorescent markers, thus providing higher sensitivity, and allowing detection of very small amounts of target biomolecules in the investigated 
solution. In addition, the nanocavities attribute peculiar spectral features to the target analytes captured by their surface, so that the presence of specific species in the solution can be inferred by a simple spectral analysis of the optical response of the read-out region. This enables parallel detection of multiple elements, thus accelerating the analysis time.

\section{Conclusions and perspectives}

This chapter details the use of $\mathrm{Si}_{3} \mathrm{~N}_{4}$ 2D-PhC nanocavities as flexible platform to realize photonic devices based on the engineering of spontaneous emission of nanoemitters in the visible spectral range. The versatility of the approach is demonstrated by coupling several types of emitters to the two photonic states allowed in a closed band gap single point defect nanocavity. In particular, DNA strands and antibodies marked with Cy3 and TRITC organic dyes have been immobilized on top of the nanocavities, while colloidal quantum dots emitting in the visible spectral range have been dropcasted on the devices and also positioned in the resonators at the maximum of the localized photonic mode. The optical measurements, carried out by $\mu \mathrm{PL}$ confocal microscopy, revealed maximum quality factors close to the theoretical estimations for all the emitters. Improvements in modeling and processing of $\mathrm{PhC}$ structures in Silicon Nitride, which is highly compatible with both biological materials and inorganic quantum emiters, let us envision a broader application of two-dimensional PhC nanocavities also in the visible spectral range. In particular, the coupling of a single colloidal dot-in-rod nanocrystal with a photonic crystal cavity would be an important milestone to reach in next years, and would allow further improvements of single photon rate and stability.

\section{Author details}

T. Stomeo ${ }^{1}$, A. Qualtieri ${ }^{1}$, F. Pisanello ${ }^{1,2}$, L. Martiradonna ${ }^{1}$, P.P. Pompa ${ }^{1}$, M. Grande ${ }^{3}$, D'Orazio $^{3}$ and M. De Vittorio ${ }^{1,4,5}$

1 Center for Bio-Molecular Nanotechnology UniLe, Istituto Italiano di Tecnologia, Arnesano (Lecce), Italy

2 Center for Neuroscience and Cognitive Systems UNITN, Italian Institute of Technology, Rovereto (TN), Italy

3 Dipartimento di Elettrotecnica ed Elettronica, Politecnico di Bari, Bari, Italy

4 National Nanotechnology Laboratory (NNL), Istituto di Nanoscienze-CNR, Via Arnesano, Lecce, Italy

5 Dipartimento Ingegneria dell'Innovazione, Università del Salento, Lecce, Italy 


\section{References}

[1] Tanabe T., Notomi M., Mitsugi S., Shinya A., Kuramochi E. All-optical switches on silicon chip realized using photonic crystal nanocavities. Applied Physics Letters 2005; 87(15) 151112.

[2] Van Laere F., Stomeo T., Cambournac C., Ayre M., Brenot R., Benisty H., Roelkens G., Krauss T.F., Van Thourhout D., Baets R. Nanophotonic Polarization Diversity Demultiplexer Chip. J. Lightwave Technology 2009; 27 417-425.

[3] Stomeo T., Van Laere F., Ayre M., Camburnac C., Benisty H., Van Thourhout D., Baets R., Krauss T.F. Integration of grating couplers with a compact photonic crystal demultiplexer on an InP membrane. Optics Letters 2008; 33(8) 884-886.

[4] Stomeo T., Grande M., Rainò G., Passaseo A., D'Orazio A., Cingolani R., Locatelli A., Modotto D., De Angelis C., De Vittorio M. Optical filter based on two coupled PhC GaAs-membranes. Optics Letters 2010; 35 (10) 411-413.

[5] D'Orazio A., De Sario M., Marrocco V., Petruzzelli V., Prudenzano F. Photonic crystal drop filter exploiting resonant cavity configuration. IEEE Transactions on Nanotechnology 2008; 7 (1) 10-13.

[6] Makarova M., Vuckovic J., Sanda H., Nishi Y. Silicon based photonic crystal nanocavity light emitters. Applied Physics Letters 2006; 89221101.

[7] Shambat G., Rivoire K., Lu J., Hatami F., Vuckovic J. Tunable-wavelength second harmonic generation from $\mathrm{GaP}$ photonic crystal cavities coupled to fiber tapers. Optics Express 2010; 18 12176-12184;

[8] Corcoran B., Monat C., Grillet C., Moss D. J., Eggleton B. J., White T. P., O'Faolain L., Krauss T. F. Green light emission in silicon through slow-light enhanced thirdharmonic generation in photonic-crystal waveguides. Nature Photonics 2009; 3 206-210.

[9] Choi Y.-S., Hennessy K., Sharma R., Haberer E., Gao Y., DenBaars S. P., Nakamura S., $\mathrm{Hu}$ E. L., Meier C. GaN blue photonic crystal membrane nanocavities. Applied Physics Letters 2005; 87243101.

[10] Rivoire K., Faraon A., Vuckovic J. Gallium phosphide photonic crystal nanocavities in the visible. Applied Physics Letters 2008; 93063103.

[11] Martiradonna L., Carbone L., Tandaechanurat A., Kitamura M., Iwamoto S., Manna L., De Vittorio M., Cingolani R., Arakawa Y. Two-dimensional photonic crystal resist membrane nanocavity embedding colloidal dot-in-a-rod nanocrystals. Nano Letters 2008; 8 (1) 260-264.

[12] Gong Y., Vucœković J. Photonic crystal cavities in silicon dioxide. Applied Physics Letters 2010; 96031107. 
[13] Grande M., O'Faolain L., White T. P., Spurny M., D'Orazio A., Krauss T. F. Optical filter with very large stopband (approximate to $300 \mathrm{~nm}$ ) based on a photonic-crystal verticaldirectional coupler. Optics Letters 2009; 34 (21) 3292-3294.

[14] Yoon D.H., Yoon S.G., Kim Y.T. Refractive index and etched structure of silicon nitride waveguides fabricated by PECVD. Thin Solid Films 2007; 515 (12) 5004-5007.

[15] Mui D. S. L., Liaw H., Demirel A. L., Strite S. and Morkoç H. Electrical characteristics of Si sub $3 \mathrm{~N}$ sub 4 /Si/GaAs metal-insulator-semiconductor capacitor. Applied Physics Letters 1991; 59 2847-2849.

[16] Chen A., Young M., Li W., Ma T.P., Woodall J.M. Metal-insulator-semiconductor structure on low-temperature grown GaAs. Applied Physics Letters 2006; 89233514.

[17] Gao H., Luginbühl R., Sigrist H. Bioengineering of Silicon-Nitride. Sensor and Actuator B, Chemical 1997; 38 (1-3) 38-41.

[18] Diao J., Ren D., Engstrom J. and Lee K. A surface modification strategy on silicon nitride for developing biosensors. Analytical Biochemistry 2005; 343 (2) 322-328.

[19] Dauphas S., Ababou-Girard S., Girard A., Le Bihan F., Mohammed-Brahim T., Vié V., Corlu A., Guguen-Guillouzo C., Lavastre O. and Geneste F. Stepwise functionalization of SiNx surfaces for covalent immobilization of antibodies. Thin Solid Films 2009; 517 6016-6022.

[20] Barth M., Kouba J., Stingl J., Löchel B., Benson O. Modification of visible spontaneous emission with silicon nitride photonic crystal nanocavities. Optics Express 2007; 15 (25) 17231-17240.

[21] Barth M., Nüsse N., Stingl J., Löchel B. and Benson O. Emission properties of high-Q silicon nitride photonic crystal heterostructure cavities. Applied Physics Letters 2008; 93021112.

[22] Eichenfield M., Camacho R., Chan J., Vahala K. and Painter O. A picogram-and nanometer-scale photonic-crystal optomechanical cavity. Nature 2009; 459 (7246) 550 555.

[23] Gong Y., Makarova M., Yerci S., Li R., Stevens M., Baek B., Nam S., Dal Negro L. and Vuckovic J. Observation of transparency of erbium-doped silicon nitride in photonic crystal nanobeam cavities. Optics Express 2010; 18 13863-13873.

[24] Khan M., Babinec T., McCutcheon M., Deotare P. and Loncœar M. Fabrication and characterization of high-quality-factor silicon nitride nanobeam cavities. Optics letters $2011 ; 36$ (3) 421-423.

[25] Pisanello F., Martiradonna L., Pompa P. P., Stomeo T., Qualtieri A., Vecchio G., Sabella S., De Vittorio M. Parallel and high sensitive photonic crystal cavity assisted readout for DNA-chips. Microelectronic Engineering 2010; 87 (5-8) 747-749.

[26] Qualtieri A., Pisanello F., Grande M., Stomeo T., Martiradonna L., Epifani G., Fiore A., Passaseo A., De Vittorio M. Emission control of colloidal nanocrystals embedded in 
$\mathrm{Si}_{3} \mathrm{~N}_{4}$ photonic crystal $\mathrm{H} 1$ nanocavities. Microelectronic Engineering 2010; 87 (5-8) 1435-1438.

[27] Martiradonna L., Pisanello F., Stomeo T., Qualtieri A., Vecchio G., Sabella S., Cingolani R., De Vittorio M., Pompa P. P. Spectral tagging by integrated photonic crystal resonators for highly sensitive and parallel detection in biochips. Applied Physics Letters 2010; 96113702.

[28] Qualtieri A., Morello G., Spinicelli P., Todaro M. T., Stomeo T., Martiradonna L., Giorgi M., Quélin X., Buil S., Bramati A., Hermier J.-P., Cingolani R., De Vittorio M. Nonclassical emission from single colloidal nanocrystals in a microcavity: a route towards room temperature single photon sources. New Journal of Physics 2009; 11033025.

[29] Le Thomas N., Woggon U., Schöps O., Artemyev M., Kazes M. and Banin, U. Cavity quantum electrodynamic (QED) with Semiconductor Nanocrystals. Nano Letters 2006; 6 (3) $557-561$.

[30] Kitamura M., Iwamoto S. and Arakawa Y. Enhanced light emission from an organic photonic crystal with a nanocavity. Applied Physics Letters 2005; 87151119.

[31] Bennett C., Brassard G.et al., Proceedings of IEEE International Conference on Computers, Systems and Signal Processing, vol. 175 (Bangalore, India, 1984).

[32] Yee K. Numerical solution of inital boundary value problems involving maxwell's equations in isotropic media. IEEE Transactions on Antennas and Propagation 1966; 14 (3) 302-307.

[33] Tandaechanurat A., Iwamoto S., Nomura M., Kumagai N. and Arakawa Y. Increase of Q-factor in photonic crystal H1-defect nanocavities after closing of photonic bandgap with optimal slab thickness. Optics Express 2008; 16 448-455.

[34] Larqué M., Karle T., Robert-Philip I. and Beveratos A. Optimizing H1 cavities for the generation of entangled photon pairs. New Journal of Physics 2009; 11 (3) 033022.

[35] Painter O., Srinivasan K., O'Brien J., Scherer A. and Dapkus P. Tailoring of the resonant mode properties of optical nanocavities in two-dimensional photonic crystal slab waveguides. Journal of Optics A: Pure and Applied Optics 2001; 3(6) S161-S170.

[36] Frédérick S., Dalacu D., Aers G., Poole P., Lapointe J. and Williams R. Optical characterisation of InAs/InP quantum dot photonic cavity membranes. Physica E: Lowdimensional Systems and Nanostructures 2006; 32 (1-2) 504-507.

[37] Hennessy K., Högerle C., Hu E., Badolato A. and Imamogølu A. Tuning photonic nanocavities by atomic force microscope nano-oxidation. Applied Physics Letters 2006; 89041118.

[38] Akahane Y., Asano T., Song B. and Noda S. Fine-tuned high-Q photonic-crystal nanocavity. Optics Express 2005; 13 (4) 1202-1214.

[39] Srinivasan K. and Painter O. Momentum space design of high-Q photonic crystal optical cavities. Optics Express 2002; 10 (15) 670-684. 
[40] Vuckovic J., Loncar M., Mabuchi H. and Scherer A. Optimization of Q-factor in photonic crystal microcavities. IEEE Journal of Quantum Electronics 2002; 38 (7) 850856.

[41] Pisanello F., De Vittorio M. and Cingolani R. Modal selective tuning in a photonic crystal cavity. Superlattices and Microstructures 2010; 47 (1) 34-38.

[42] Krauss T. F. K. Photonic crystals: Cavities without leaks. Nature Materials 2003; 2 (12) 777-778.

[43] Carbone L., Nobile C., Giorgi M. D., Sala F., Morello G., Pompa P.P., Hytch M., Snoeck E., Fiore A., Franchini I. R., Nadasan M., Silvestre A. F, Chiodo L., Kudera S., Cingolani R., Krahne R. and Manna L. Synthesis and micrometer-scale assembly of colloidal CdSe/ CdS nanorods prepared by a seeded growth approach. Nano Letters 2007; 7 2942-2950.

[44] Pisanello F., Qualtieri A., Stomeo T., Cingolani R., Martiradonna L., Bramati A. and De Vittorio M. High-Purcell-factor dipolelike modes at visible wavelengths in H1 photonic crystal cavity. Optics Letters 2010; 35 (10) 1509-1511.

[45] Rico-Garcia J., Lopez-Alonso J. and Alda J. SPIE 2005: Multivariate analysis of photonic crystal microcavities with fabrication defects: Proceeding of SPIE Europe, 9-11 May 2005, Sevilla, Spain.

[46] Andreani L., Panzarini G. and Gérard J. M. Strong-coupling regime for quantum boxes in pillar microcavities: Theory. Physical Review B 1999; 6013276.

[47] Adawi, A. \& Lidzey, D. A design for an optical-nanocavity optimized for use with surface-bound light-emitting materials. New Journal of Physics 2008; 10065011.

[48] Ganesh N., Zhang W., Mathias P., Chow E., Soares J., Malyarchuk V., Smith A. and Cunningham B. Enhanced fluorescence emission from quantum dots on a photonic crystal surface. Nature Nanotechnology 2007; 2 (8) 515-520.

[49] Skivesen N., Têtu A., Kristensen M., Kjems J., Frandsen L. H. and Borel P. I. Photoniccrystal waveguide biosensor. Optics Express 2007; 15 (6) 3169-3176.

[50] Li M., He F., Liao Q., Liu J., Xu L., Jiang L., Song Y., Wang S. and Zhu D. Ultrasensitive DNA detection using photonic crystals. Angewandte Chemie 2008; 120 (38) 7368-7372.

[51] Fan J., Chee M. and Gunderson K. Highly parallel genomic assays. Nature Reviews Genetics 2006; 7 (8) 632-644.

[52] Schena M., Shalon D., Davis R. and Brown P. Quantitative monitoring of gene expression patterns with a complementary DNA microarray. Science 1995; 270 (5235) 467-470.

[53] Spurgeon S., Jones R., and Ramakrishnan R. High throughput gene expression measurement with real time PCR in a microfluidic dynamic array. PLoS One 2008; 3 e1662.

[54] Purcell E., Torrey H. and Pound R. Resonance Absorption by Nuclear Magnetic Moments in a Solid. Physical Review 1946; 69 (1-2) 37-38. 
[55] Noda S., Fujita M. and Asano T. Spontaneous-emission control by photonic crystals and nanocavities. Nature Photonics 2007; 1 449-458.

[56] Painter O., Lee R., Scherer A., Yariv A., O’brien J., Dapkus P. and Kim I. Two-dimensional photonic band-gap defect mode laser. Science 1999; 284 (5421)1819-1821.

[57] Fan S. and Joannopoulos J. Analysis of guided resonances in photonic crystal slabs. Physical Review B 2002; 65235112.

[58] Crozier K., Lousse V., Kilic O., Kim S., Fan S. and Solgaard O. Air-bridged photonic crystal slabs at visible and near-infrared wavelengths. Physical Review B 2006; 73 115126. 
